\title{
Laser Epilation for Unwanted Hair in the Larynx
}

Min Woo Park

Seung-Kuk Baek

Kwang-Yoon Jung

Department of Otorhinolaryngology-Head and Neck Surgery, Korea University College of Medicine, Seoul, Korea
Received November 12, 2012

Reviced November 26, 2012

Accepted November 30, 2012

\section{Correspondence}

Kwang-Yoon Jung

Department of Otorhinolaryngology-Head and Neck Surgery, Korea University College of Medicine, Anam-dong 5-ga 126-1, Seongbuk-gu, Seoul 136-705, Korea

Tel: +82-2-920-5486

Fax: $+82-2-925-5233$

E-mail: kyjungdakumc.or.kr

(C) Korean Society for Laser Medicine and Surgery

(c) This is an open access article distributed under the terms of the Creative Commons Attribution NonCommercial License (http://creativecommons.org/ licenses/by-nc/3.0) which permits unrestricted noncommercial use, distribution, and reproduction in any medium, provided the original work is properly cited.
Variable skin flaps are used in reconstruction of defects following surgical resection of larynx cancers. Skin taken from distant sites often contains hair-bearing skin, which can create a problem following reconstruction. In particular, patients with hairy laryngeal flaps often complain of irritation, voice change, and trapping of food. We report on the removal of hair from skin flap of the larynx using a diode laser.

\section{Key words}

Hair; Laser; Reconstruction; Larynx cancer; Complication 


\section{INTRODUCTION}

A variety of the flaps used to reconstruct defects of the head and neck region following surgery for malignant disease. The flaps which contain hair follicles may result in unwanted hair growth and can cause significant distress with potential psychosocial consequences. Hair removal with laser has become a popular treatment that is considered the most efficient method for the reduction of unwanted hair. ${ }^{1}$ There are several reports that laser hair removal following reconstructive surgery in oral cavity, pharynx, and esophagus. ${ }^{2-6}$ However, there is no report for the use of laser treatment for postoperative unwanted hair of the larynx in our best knowledge. We report a case of significant laryngeal hair growth which was managed with diode laser.

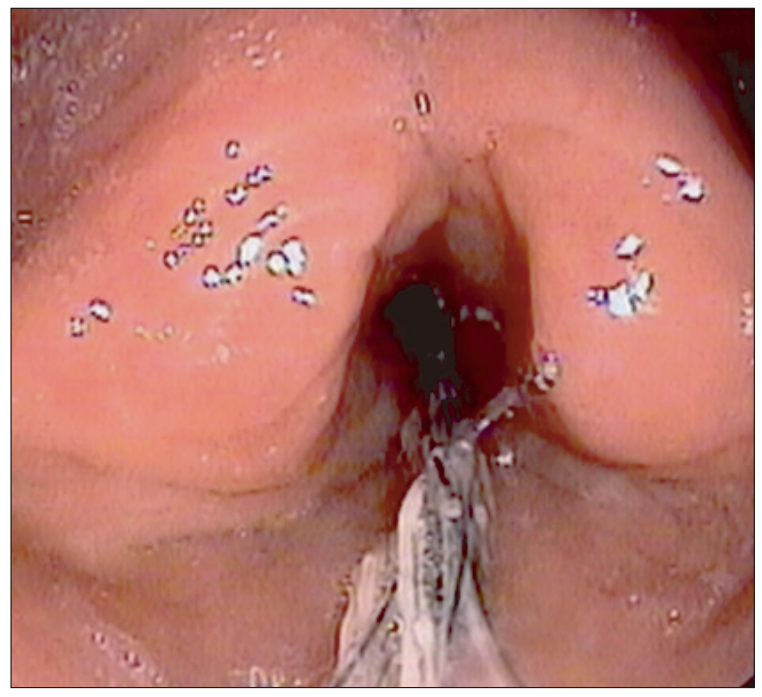

Fig. 1. Hair growth in the larynx following reconstruction.

\section{CASE REPORT}

A 70-year-old man underwent extended vertical hemilaryngectomy with glottic squamous cell carcinoma. The defect of larynx was reconstructed with skin graft from the neck. The graft took well and healing was uneventful. After several months the graft had begun to grow hair very extensively in the larynx (Fig. 1). He presented the voice change, tickling, gagging and swallowing difficulty. A $980 \mathrm{~nm}$ diode laser (Biolitec AG, Jena, Germanyl was used for the hair removal procedure. The laser was delivered to larynx through transnasal flexible laryngoscope. All procedures were performed in unsedated patients with local anesthesia while the patient was seated in a standard ENT examining chair. The nose is prepared with two nasal sprays with $2 \%$ lidocaine and $0.5 \%$ phenylephedrine applied to each nostril. The larynx was anesthetized with $2 \mathrm{ml}$ of $2 \%$ lidocaine which was directly administered to the larynx through the flexible laryngoscope. His symptoms have improved after laser hair removal. Laser hair epilation has been performed at intervals of 1 or 2 months. The progressive improvement in hair reduction was found during the course of nineteen treatments (Fig. 2). No adverse effects occurred during the treatment.

\section{DISCUSSION}

Although not a severe postoperative complication, unwanted hair growth following flap reconstruction can cause patient distress. Our case showed that the use of skin graft taken from hair-bearing skin without previous ablation of hair can lead to the undesired side effect of hair. The transferred hair-bearing skin can show more profound hair growth than at the donor site, resulting

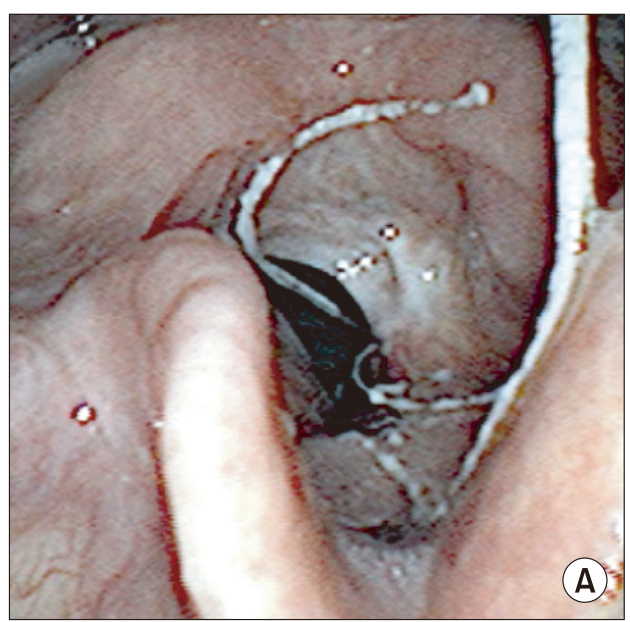

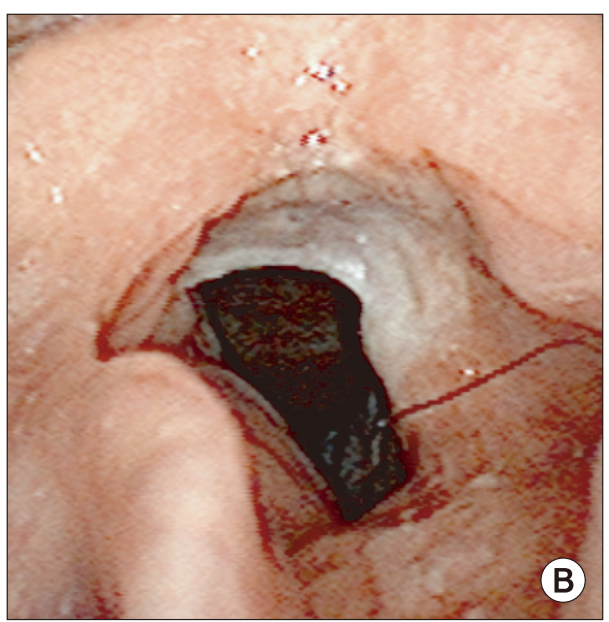

Fig. 2. The progressive reduction of hair during the course of laser treatments. (A) The larynx after 10 times of laser treatment shows reduction of hair. (B) The larynx after 19 times of laser treatment shows much reduced hair. 
from flap revascularization stimulation. ${ }^{7}$ For prevention of unwanted hair growth, preoperative flap selection for hair-free skin is important and preoperative flap depilation should be performed when hair-bearing skin is used for flap reconstruction.

Recently, unwanted hair after flap reconstruction has been treated in variable methods. The trimming of hairs under endoscopic guidance is well documented, but it is often time consuming and need the many hospital stays. ${ }^{8,9}$ Electrolysis before flap transfer can also be timeconsuming, technically difficult. Electrolysis has been used with partial success to manage an intraoral hairy rectus abdominis myocutaneous free flap. ${ }^{5}$ The complete hair depilation has been reported with postoperative radiotherapy but the result is unpredictable and prolonged staged reconstruction may preclude the use of postoperative radiotherapy. ${ }^{10}$

The use of laser hair removal for unwanted hair of reconstructed flap has been discussed by Kuriloff et al. ${ }^{2}$ They describe the use of $\mathrm{CO}_{2}$ laser for depilation of the hypopharynx following flap reconstruction. They describe the targeting of individual hair follicles using microscope under the general anesthesia.

Laser hair epilation with Nd:YAG was recently reported for the management of an intraoral hairy flap. ${ }^{3,6}$ The Alexandrite laser has offered good results. ${ }^{4}$ Until now, the laser application for hairy flap is limited mainly in the oral cavity due to accessibility. For cases of hypopharynx and esophagus, general anesthesia was necessary for hair removal. We, for the first time, report that laser treatment of unwanted hair in the larynx using transnasal flexible endoscope without general anesthesia. Transnasal flexible endoscope is a safe and well-tolerated procedure that may be performed in a procedure room in the outpatient or day-care setting. The laser application through flexible fiber allows the access of laser to the larynx.

For successful laser treatment without complication, laser requires an effective fluence, appropriate spot size for the area being treated. The number of laser treatments is often dependent on the amount, thickness and site of hair. Hair in different areas of the body has the variable length of growth cycles. Multiple laser treatments are necessary because laser only targets follicles in the anagen phase. ${ }^{11}$ Our case also needed multiple treatments for hair reduction.

Laser depilation of unwanted hair in the larynx with diode laser is effective and beneficial. Treatment alleviates complaints of voice and swallowing. Laser treatment using transnasal flexible laryngoscope may be appropriate method for hair removal in the larynx following reconstructive laryngeal surgery.

\section{REFERENCES}

1. Hovenic W, DeSpain J. Laser hair reduction and removal. Facial Plast Surg Clin North Am 2011;19:325-33.

2. Kuriloff DB, Finn DG, Kimmelman CP. Pharyngoesophageal hair growth: the role of laser epilation. Otolaryngol Head Neck Surg 1988;98:342-5.

3. Lumley $\mathrm{C}$. Intraoral hair removal on skin graft using $\mathrm{Nd}$ :YAG laser. Br Dent J 2007;203:141-2.

4. Conroy FJ, Mahaffey PJ. Intraoral flap depilation using the longpulsed alexandrite laser. J Plast Reconstr Aesthet Surg 2009; 62:e421-3.

5. Hall RR, Pearce DJ, Brown T, McMichael AJ. Unwanted palatal hair: a consequence of complex oropharyngeal reconstruction. J Dermatolog Treat 2009;20:149-51.

6. Shim TN, Abdullah A, Lanigan S, Avery C. Hairy intraoral flap-an unusual indication for laser epilation: a series of 5 cases and review of the literature. Br J Oral Maxillofac Surg 2011;49:e502.

7. Conley JJ. Skin flap and skin graft complications. In: Conley JJ, editor. Complications of Head and Neck Surgery. Philadelphia: WB Saunders Co.; 1979. p.457.

8. McLean G, Laufer I. Hairy esophagus: a complication of pharyngoesophageal reconstructive surgery in two cases. AJR Am J Roentgenol 1979;132:269-70.

9. Kwak DL, Kattan KR. Hair growing in the esophagus: complication of reconstruction of the pharynx and esophagus. South Med J 1983;76:544-5.

10. Maisel RH, Liston SL, Adams GL. Complications of pectoralis myocutaneous flaps. Laryngoscope 1983;93:928-30.

11. Haedersdal M, Gøtzsche PC. Laser and photoepilation for unwanted hair growth. Cochrane Database Syst Rev 2006;(4): CD004684. 